INTERNATIONAL JOURNAL OF SYSTEMATIC BACTERIOLOGY

Vol. 17 , No. 3 July 1967

pp. $281-314$

Copyright 1967, Iowa State University Press

\title{
INTERNATIONAL COMMITTEE ON NOMENCLATURE OF BACTERIA SUBCOMMITTEE ON STREPTOCOCCI AND PNEUMOCOCCI
}

\author{
Minutes of the Meeting Held in Moscow \\ July 21,1966
}

I. Introductory items

A. The meeting was held in the Streptococcus Department of the Gamaleya Institute from 11:00 a. m. to 5:00 p. m. July 21, 1966. Chairman Williams presided and welcomed the following members: Colman, Guthof, Hansen, Kobler, Lyampert, Mocquot, Moody, Ottens, Parker, Rotta, Sharpe, Updyke, and Wahl.

B. The chairman expressed appreciation to Dr. Lyampert and her staff for making arrangements for the meeting at the Gamaleya Institute.

C. Chairman Williams regretfully announced the deaths of the following members since the Montreal meeting: Dr. A. T. Wilson, Dr. P. M. F. Shattock, and Dr. C. Baldovin-Agapi.

D. The following appointments to the Subcommittee were announced: Dr. Heeschen, Kiel, Germany; Dr. Robert Austrian, Philadelphia, Pennsylvania; Dr. I. M. Lyampert, Moscow, U.S.S. R. ; Dr. Hutton Slade, Chicago, Illinois. Dr. Elizabeth Sharpe, Reading, England; and Dr. Max D. Moody, Atlanta, Georgia. Dr. Moody was also appointed to serve as Secretary upon the death of Dr. Wilson.

E. The resignation of Dr. F. Kauffmann, Copenhagen, was announced.

F. The meeting proceeded according to a previously arranged agenda. 
II. List of Agenda, discussion, and actions taken at the meeting

A. Group A Streptococci

1. Designation of M-types

a. Criteria for designation of new M-types

Dr. Moody indicated that it becomes increasingly apparent that guidelines for designation of new $M$-types of Group A streptococci are needed and that official recognition should be made at the appropriate time. As new M-types are dis covered by investigators, it is necessary to show that specified criteria have been met before a number if assigned in order that confusion in the literature be kept to a minimum.

Action: Considerable discussion of the topic lead to the following consensus:

(1) The Subcommittee should assume the responsibility for studying and recognizing new M-types of Streptococcus pyogenes and to recommend that the Judicial Commission or the Executive Committee of the ICNB adopt the recommended numerical designation for the newly discovered M-type.

(2) Strains proposed as new $M$-types may be recognized as provisional $M-t y p e s$ and described by the investigator according to his wishes until the Subcommittee has determined independently that the strain meets necessary criteria for numerical designation and until a number has been adopted.

(3) Provisional types should be submitted to one of the following Streptococcus reference laboratories for comprehensive study: Colindale (Dr. Parker); Prague (Dr. Rotta); Atlanta (Dr. Moody); or Rockefeller University (Dr. Lancefield). The laboratory to which the culture is first sent should arrange for studies to be made in at least two reference laboratories which may or may not include the receiving laboratory. The Secretary should be notified of such requests 
and, if desired, asked to make the arrangements for examination of the strain.

(4) Data obtained by the reference laboratories which supports or contraindicates designation of a number for a new M-type should be submitted to the Secretary. The Secretary will notify members of the Subcommittee of the findings. The executive Committee of the ICNB will be asked to approve the recommendations of the Subcommittee even between Congresses if indicated and to give permission for publishing the report in the International Journal of Systematic Bacteriology where possible.

(5) It was recognized that certain strains may bear characteristics indicating the presence of $\mathrm{M}$-protein of more than one $M-t y p e$ (e.g. provisional type 5l). In such cases, a type number still can be assigned but with an indication that such properties exist.

b. Status of type 51

Some Subcommittee membërs have inquired about the status of type 51. At the Montreal meeting, it was proposed that "5l" be designated for strains described by Wilson and Wiley. No official action was taken, however. Subsequently, Dr. Wiley (personal communication) has indicated that mo "recent and unpublished work with double diffusion experiments in agar gel has confirmed earlier observations." These tests show that the two $M$ antigens, 14 and $51, "$... are entirely distinct substances that diffuse separately in agar and have completely different immunological specificity, giving a pattern of non-identity."

Action: It was proposed that type "5l" receive official designation by the Subcommittee. The matter was discussed, and the consensus indicated that type 51 should be considered a provisional type until the reference laboratories have had an opportunity to study the strains adequately.

c. Proposal to designate type 35 strains as type 49

The following proposal of Dr. Lancefield was read by the Secretary: "... on the basis of the findings of Dr. Grove T Wiley and Dr. Franklin H. Top, Jr. that Type 35 be 
dropped from the roster of Group A types and that the two known strains ( $\mathrm{Cl} 71$ and $\mathrm{Cl}$ 35) be transferred to Type 49. This is based on the finding that the Type 35 strains conform to the characteristics which these investigators and others have attributed to Type 49: M production, as determined by precipitin reactions and bactericidal tests, possession of $T$ antigen in common $(T-14)$, and production of lipoproteinase (unpublished findings of Dr. Franklin H. Top, Jr. )."

Dr. Parker outlined evidence (Appendix 1, by Fraser, Maxted, and Parker) in their studies with types 14, 35, 49, and 51 , which indicate that types 35 and 49 are the same $\mathrm{M}$-protein; they also believe that these " $\mathrm{T}-14$ " strains belong to type 49 .

Action: The proposal of Dr. Lancefield was approved by the Subcommittee. The Secretary will notify the Secretary of the Section on Culture Collections and arrange for publishing a note of the action in the International Journal of Systematic Bacteriology.

\section{d. Proposal for designation of Type 52}

Dr. Lancefield's proposal was read as follows: ". . that the designation as $T$ ype 52 should be assigned to Group $A$ strains studied by Dr. Franklin $\mathrm{H}$. Top, Jr. He has prepared antisera against strains which he has found in the current studies from the Department of Pediatrics, University of Minnesota Medical School, being carried out at the Red Lake Indian Reservation. These strains have the $T$ antigen designated $3,13, B 3264$ and give very good $M$ precipitin and bactericidal reactions with the serum prepared by Dr. Top.

"Strains giving those reactions have been received by $\mathrm{Dr}$. Top from Mr. Maxted, and I have identified two additional Btrains isolated by Dr. Wannamaker in Minnesota prior to 1957 (when he deposited them in my collection). Dr. Top has a number of such strains in his collection. It seems to be justifiable and highly desirable to identify these as Group A, Type 52."

Action: The members felt that, in accordance with the actions described above (Item 1.a.), the type should be considered as a provisional type until reference laboratories have sufficient opportunity to study the strains. 
2. International Type Distribution Survey, 1964-1965 Dr. Parker summarized date obtained from the International Type Distribution Survey of 1964-1965. The purposes of the survey were (1) to determine whether there are major differences in the type distribution of $\underline{S}$. pyogenes in different parts of the world, and (2) to stimulate contact between laboratories practicing Streptococcus typing and to improve the reproducibility of the typing results obtained by them. Central laboratories of the following countries participated: Canada, Denmark, Hungary, Israel, Japan, Czechoslovakia, Russia, Germany, Italy, Britain, and the U.S. A. Dr. Parker's report included 12 tables of extensive data. It was recognized that supervision of the project and collation of all the data was a tremendous task. The Subcommittee is indebted to Dr. Parker, his staff, and Dr. Williams for a job well done. Most participants expressed interest in extending the survey, as it helped them to improve their typing competency.

Action: It was agreed that Dr. Parker would prepare two manuscripts describing the study and submit drafts for consideration to the participants of the study. Part I would be concerned with interrelationships between the $M$ - and T-typing systems; Dr. Parker suggests that this be left unpublished. Part II would be concerned with type distribution in various parts of the world and would be prepared for publication.

A committee consisting of Drs. Parker, Rotta, Köhler, and Moody was asked to develop a plan for continuing cooperation between laboratories practicing Streptococcus typing. The committee met in Moscow and recommended that the activities could be divided into two parts as follows: (1) Regular cooperation between National Reference Laboratories involving comparison of typing results. The aims of this project would include: (a) better possibility for control of the sera employed by the participating laboratories, (b) the achievement of a degree of standard in typing that would permit good comparability of results, (c) more efficient exchange of standard strains, reference sera and standard laboratory techniques, (d) gradual extension of the typing serum sets in the different laboratories, and (e) provision of general information on Streptococcus type prevalence in different parts of the 
world. Dr. Rotta will prepare a protocol for this coope ration and organize it in conjunction with activities of the WHO International Reference Center for Streptococcus Typing, which is located in Prague, under his direction. (2) Collation and analysis of data on streptococcal type prevalence in the world. Dr. Köhler will undertake the preparation of a plan and the organization of the study. It was suggested that a study analogous to the one Drs. Parke $r$ and $W$ illiams supervised would be repeated at three or four year intervals. The main objective would be to receive information or type distribution of streptococci in different parts of the world. The study would be carried out by a number of National Reference Laboratories. Dr. Köhler will consider the possibility of using computer analysis on the data. It is anticipated that this project will begin in 1968 .

3. World Health Organization International Reference Center for St reptococcus Typing

Dr. Rotta presented the following summary concerning the designation of the WHO center in Prague:

The Streptococcus Reference Laboratory in Prague has been designated as the WHO International Reference Centre for Streptococcus Typing. The Prague Laboratory will assist in this function on WHO programmes relating to studies on streptococcal infections and their sequellae.

In agreement with the purposes outlined by WHO, the functions of the WHO International Reference Centre should include:

(1) Maintenance of a culture collection of reference strains for the preparation of antisera and the production of extracellular products;

(2) Preparation of reference grouping and typing sera and provision of facilities for testing grouping and typing sera prepared in regional or national reference centres;

(3) Group and type identification of streptococci, the facilities for performing the grouping and typing

* Sera produced by commercial forms should be controlled by National Reference Centres. 
a limited number of streptococci sent from abroad (especially from national reference centres for checking);

(4) Work concerned with the standardization of antibody titrations;

(5) The introduction and working out of new diagnostic procedures for cultivation, identification, classification and serology, and the standardization of these techniques;

(6) Aid to national reference laboratories through training of personnel and supply of reference strains, sera, etc. ;

(7) The International Reference Laboratory should be available for assistance in the coordination of international epidemiological studies.

Close collaboration with other Streptococcus Reference Laboratories and contact with the Subcommittee on Streptococci and Pneumococci is considered essential for developing the activities of the Prague Laboratory as a WHO Centre.

4. The investigations of some extracellular products of Group A streptococci

Dr. Lyampert described work in her department conce rned with antigenic components revealed in preparations of erythro-genic toxin of different stages of purity and in crystalline proteinase (Appendix 2).

\section{The bacitracin test for recognition of Group $A$}

\section{streptococci}

Dr. Rotta described work of Jelinkova and Rotta in Prague. The following summary of Appendix 3 was prepared by Dr. Rotta:

The bacitracin test is a valuable screening method for the recognition of group A streptococci in diagnosis, especially when large numbers of strains are examined. Its reliability, however, is limited by the fact that there exist group A strains of lower bacitracin sensitivity; the sensitivity of these strains is of an equal as that of some strains of certain other serological groups. 
The bacitracin test was employed for the classification, under standard conditions, of samples of group $A, B, C$, $G$, and $L$ strains occurring among men or animals in this country at the present time.

It was found there circulates a certain number of strains of groups $C$ and $G$ whose sensitivity is analogous to the less sensitive group A strains; a comparable degree of sensitivity was also encounte red in some group $L$ strains. In this respect it was interesting to find there also exists some relationship between group L and group A strains as regards serological activity and the composition of group-specific carbohydrate.

The degree of reliability of the bacitracin test in practice depends on the one hand on its arrangement and on the other hand on the origin of the material under study.

\section{Tetracycline resistant Group A streptococci}

Dr. Parker discussed a table of data on tetracycline resistance of Group A strains studied in their laboratories. There was evidence of a great increase in percentage of resistant strains over the period 1961 to 1965.

Dr. Parker stated they have been unsuccessful in inducing tetracycline resistance in vitro.

Dr. Köhler cited his publication (Zentrbl. Bakteriol. , Parasit., Infek., Hyg., 197:461-467 (1965)) on the tetracycline sensitivity of Streptococcus pyogenes. His summary of the study is as follows: "The number of tetra* cycline-resistant strains of Streptococcus pyogenes is increasing. Investigation resuits available so far from Great Britain, Australia, and the U.S. A. report $5-32 \%$ nonsensitive strains.

"Our studies of $2575 \mathrm{~A}-\mathrm{streptococci}$ during the period November 1963 to March 1965 show $0.52 \%$ tetracyclineresistant strains. This number is increasing, since only $0.19 \%$ nonsensitive strains were observed from November 1963 to December 1964, whereas there were $1.81 \%$ during the period from January to March 1965 . The difference is significant. As shown also by other investigators, as far as they carried out typifications, strains of type 12 were predominant. A relationship between tetracycline resistance and hospital infection cannot be established." 
7. Sensitivity of Group A Streptococcus cultures to temperate phages

Dr. Leonova, a colleague of Dr. Lyampert, investigated 25 temperate streptococcal phages isolated from lysogenic cultures of Group A streptococci by UV induction (Appendix 4).

\section{B. Classification of nonhemolytic streptococci}

Mr. Colman and Dr. Williams introduced an interesting study on the use of old and new parameters, including cell-wall composition and electronic computers, to demonstrate systematic relationships of streptococci (Appendix 5). The paper was concerned mostly with 342 strains conceivably called $\underline{S}$. viridans. Two hundred sixteen strains were subjected to "cluster analysis" in a computer program. The computer was instructed to determine the similarity coefficient of each strain in relation to every other strain and then print out, at a number of different significance levels, the clusters for which this relationship is hardly due to chance; as the significance level falls the clusters contain more strains, and new but less homogeneous clusters are formed.

Discussion. The conserisus of the committee was that this approach to classification of streptococci is a good one and should be pursued. Dr. Wahl described it as "interesting and courageous."

\section{Status of "F" antigens}

Dr. Ottens described a new family of carbohydrate antigens in streptococci, originally found in Froup $F$, but present in other groups such as A, C, F, G, L, S, T, MG, and sometimes acting as the sole carbohydrate antigen. Their presence can give rise to unexpected and confusing cross-reactions. The advisability of designating the secondary, carbohydrate antigen in strains of various groups, such as A,I streptococci, C, III streptococci, etc. , was discussed.

Action: It was suggested by the Subcommittee that a set of reference strains containing the various secondary carbohydrate antigens be placed in either or both the NCTC and the ATCC. It was also agreed that the work is of great importance in the preparation of standard antisera. The Subcommittee preferred not to propose the designation of double carbohydrate antigens at this time. 
D. Streptococci used for preparation of grouping antisera Dr. Williams discussed the summarization of the identity of strains used for producing antisera for 18 streptococcal groups in 17 different laboratories (previously distributed to Subcommittee members). It became obvious that greater uniformity of strains used for producing antisera should be attempted among the laboratories.

Action: Volunteers on the committee were solicited to collect and make a study of strains used by various laboratories producing $\mathrm{g}$ rouping antisera. The volunteers were asked to make recommendations during the next four-year interim for the use of two or three strains best suited for serum production for each group. The following individuals will be responsible for the groups indicated: Dr. Rotta, A and L; Dr. Moody, B; Dr. Köhler, C and G; and Drs. Sharpe and Guthof, D. Dr. Moody will notify each of the laboratories that they will be asked to supply their strains to one of the above individuals for study.

E. Miscellaneous items

1. Taxonomic status of Pediococcus cerevisiae During the past interim, strain ATCC 8081 was studied in several laboratories to determine whether the strain should be adopted as the neotype strain of the species Pediococcus cerevisiae Balcke. The suggestion had been made by the Judicial Commission of the International Committee on Nomenclature of Bacteria that our Subcommittee submit a recommendation. The results of the studies were inconclusive and would not justify a recommendation.

Action: The Subcommittee will formulate an ad hoc committee to pursue the study of the question during the next four years. The Judicial Commission will be notified of this decision.

2. Taxonomic status of pneumococci

Dr. Updyke stated that the editors of Bergey's Manual would like the opinion of the Subcommittee on the question of the validity of the generic name Diplococcus. At the Montreal meeting, it was the consensus that D. pneumoniae be designated Streptococcus pneumoniae, but no formal action was taken. 
The question of two different systems for designating pneumococcal types in the U.S.A. and in Denmark was discussed briefly. No resolutions to the problem were offered and the subject was dropped regretfully.

Action: Dr. Updyke moved that the necessary steps be taken for the Subcommittee to make a written recommendation to the editors of Bergey's Manual that Streptococcus pneumoniae be used in the new edition instead of Diplococcus pneumoniae. There was unanimous agreement that this be done. The Secretary was directed to determine the appropriate procedure and action required to effect the recommendation.

3. Taxonomic status of "minute" streptococci Group E

Dr. Niven's request for an opinion concerning the species designation of Streptococcus anginosus (7th Ed. , Bergey's Manual) was discussed. Such strains are "minute" streptococci belonging to Lancefield's serological Group $F$ and Type I Group $G$ and comprise a relatively homogeneous group based upon their physiological characteristics.

Action: After considerable discussion, and in view of the status of " $F$ " antigens and other considerations, the Subcommittee thought it inappropriate at this time to include a single species of Streptococcus organisms containing more than one Lancefield group antigen.

No action was taken on Dr. Niven's suggestion that Group $E$ streptococci be given a species designation, since no informative report was available.

4. Availability of W.H. O. reprints

Dr. Updyke indicated that Columbia University Press no longer has reprints of W.H. O. reports on streptococci, pneumococci, etc. She would like a mechanism for making them available.

Action: It was recommended that NCDC investigate the possibility of making them available from the NCDC.

5. Subcommittee membership

The Subcommittee recognized the desirability of geographical representation. Lack of representation from South East Asia, Japan, South Africa, South America, 
and Australia was noted. The name of Dr. Kodama, from Japan, was proposed for membership. Subcommittee members were aware of no active workers in the field of Streptococcus or Diplococcus in South Africa, South America, or Australia. The names of Dr. Deibel (USA) and Dr. J. Szita (Hungary) were proposed for membership. A copy of current membership is listed in Appendix 6 .

6. Future meetings

The next meeting will be held in Mexico City in 1970.

7. Adjournment

The Subcommittee adjourned at 5:00 p. m.

Max D. Moody, Secretary

\section{APPENDIX 1}

\section{Agglutination patterns in types 14 and 49}

C. A. M. Fraser, W.R. Maxted, and M. T. Parker

Central Public Health Laboratory

Streptococcus Laboratory, Colindale, London

Since the Red Lake glomerulonephritis epidemic in 1955 , type 49 has not been reported from any similar incident. Anti-M sera for this type seem generally to be poor, and this may account for the apparent rarity of the type.

Feasby (1944), de Moor (1960) and Sramék (1964) have all reported outbreaks of glomerulonephritis as sociated with strains which agglutinate only with sera for type 14. We have examined the Dutch and Czech strains, and have also found a large number of similar cultures in two studies of streptococcal skin infection in areas where there was a high incidence of glomerulonephritis. A collection of cultures isolated from skin infections in Baltimore (see Markowitz et al. 1965) also included some more.

Köhler in 1963 showed that types $14,35,49$ and 51 all had a common agglutinating antigen, and we confirmed this. We made sera with the type strains of types 14 and 49 , and also 